\title{
Incidence Rates of Bleeding and Emergency Surgery Due to Trauma or Fracture Among Japanese Patients with Non-valvular Atrial Fibrillation Receiving Oral Anticoagulation Therapy
}

\author{
Masahiro Yasaka - Hiroyuki Yokota - Michiyasu Suzuki • \\ Teiichi Yamane $\cdot$ Yasuhisa Ono
}

Received: March 26, 2020 / Published online: May 11, 2020

(C) The Author(s) 2020

\begin{abstract}
Introduction: In patients with nonvalvular atrial fibrillation (NVAF) receiving oral anticoagulants (OACs), reversal of coagulopathy can be achieved with specific reversal drugs such as idarucizumab, which is indicated for use in patients treated with dabigatran for cases of lifethreatening or uncontrolled bleeding or during emergency procedures that may cause serious bleeding. This study examined the incidence rate (IR) of emergency surgeries and major bleeding episodes associated with fractures and trauma in Japanese patients with NVAF receiving OACs.
\end{abstract}

Digital Features To view digital features for this article go to https://doi.org/10.6084/m9.figshare.12130329.

M. Yasaka $(\square)$

Department of Cerebrovascular Medicine and

Neurology, National Hospital Organization Kyushu

Medical Center, 1-8-1 Jigyohama, Chuo-ku,

Fukuoka 810-8563, Japan

e-mail: Yasaka@kyumed.jp

\section{H. Yokota}

Department of Emergency and Critical Care

Medicine, Nippon Medical School, 1-1-5 Sendagi,

Bunkyo-ku, Tokyo 113-8603, Japan

M. Suzuki

Department of Neurosurgery, Yamaguchi University

School of Medicine, 1077-1 Yoshida, Yamaguchi-

City, Yamaguchi 753-0841, Japan
Methods: This retrospective, non-interventional analysis of health insurance claims was conducted using data from 62,888 OAC-naive adult patients with NVAF who initiated dabigatran, warfarin, apixaban, rivaroxaban, or edoxaban between March 2011 and June 2016. The primary endpoint was overall IR of emergency surgery or major bleeding due to fracture or trauma.

Results: Overall IR of emergency surgery or major bleeding due to fracture or trauma considering outcomes until OAC discontinuation was 0.489 per 100 patient-years (PY) (95\% confidence interval [CI] 0.406-0.572). Considering first OAC exposure only, the IR was 0.483 per 100 PY (95\% CI 0.394-0.573). Emergency surgery/major bleeding events due to fracture or trauma was highest in those aged $\geq 75$ years (0.611 per 100 PY [95\% CI 0.481-0.741]).

\section{T. Yamane}

Department of Cardiology, Jikei University School of Medicine, 3-25-8, Shinbashi, Nishishinbashi, Minato-ku, Tokyo 105-8461, Japan

\section{Y. Ono}

Nippon Boehringer Ingelheim, 2-1-1 Osaki, Shinagawa-ku, Tokyo 141-6017, Japan 
Conclusions: Fewer than one in 200 patients per year with NVAF receiving OACs experience emergency surgeries and major bleeding episodes associated with fractures and trauma; however, the IR of these events is markedly higher in patients of advanced age.

Trial registration: ClinicalTrials.gov 207, NCT03254147.

\section{PLAIN LANGUAGE SUMMARY}

Patients with an abnormal heart rhythm (nonvalvular atrial fibrillation [NVAF]) have a higher risk of blood clots and stroke (which is when the blood supply to part of the brain is blocked). To reduce these risks, patients can take anticoagulants that slow or prevent the formation of blood clots. However, if the patient needs major emergency surgery or has a severe injury, the anticoagulants can increase their risk of bleeding, which can sometimes be life-threatening. There are drugs (e.g., idarucizumab) that can be used to reverse the effects of anticoagulants in this type of emergency. What we don't know is how many NVAF patients in Japan who are on anticoagulants have emergency surgeries or major bleeding after an injury, and therefore may require a reversal drug. The authors looked at Japanese health insurance claim data from 62,888 adult patients with NVAF who started taking an anticoagulant. They found that, annually, approximately $0.5 \%$ of the patients had emergency surgery or a major bleed associated with a fracture or injury. In very elderly patients (aged at least 75 years), the annual percentage was approximately $0.6 \%$, which was almost double the annual percentage in patients aged less than 65 years. The authors concluded that, even though the number of people requiring a reversal agent are quite small, it is important to have an effective reversal agent for patients on anticoagulants, particularly older patients.

Keywords: Idarucizumab; Major bleeding; NVAF; Oral anticoagulant; Surgery

\section{Key Summary Points}

Why carry out this study?

Patients who take oral anticoagulants (OACs) and require emergency surgery or experience medical trauma may need rapid reversal of the anticoagulant effect to reduce bleeding risks.

To assess the need for reversal agents, we determined the incidence of major surgeries or major bleeding associated with fractures and trauma using data from 62,888 OAC-naive Japanese patients with nonvalvular atrial fibrillation (NVAF) who initiated OACs

\section{What was learned from this study?}

The overall incidence rate (IR) of emergency surgery or major bleeding due to fracture or trauma was 0.489 per 100 patient-years.

The IR in patients aged $\geq 75$ years was almost double that in patients aged $\leq 64$ years $(0.611$ and 0.317 per 100 patientyears, respectively).

Although the IR was quite low, this study highlights the importance of having an effective reversal agent for patients on OACs, particularly older patients.

\section{INTRODUCTION}

Nonvalvular atrial fibrillation (NVAF) is a major risk factor for thromboembolism and increased incidence of stroke in affected individuals $[1,2]$. While the oral anticoagulant (OAC) warfarin, a vitamin $\mathrm{K}$ antagonist (VKA), prevents stroke in patients with NVAF $[1,3]$, it increases the risk of hemorrhage and adverse events due to its drug-drug interactions, food-drug interactions, and variable patient response via genetic polymorphisms [4-6]. Direct OACs (DOACs) have been developed to overcome these limitations 
[7], and include dabigatran, rivaroxaban, apixaban, and edoxaban [8]. Patients taking DOACs who undergo major emergency surgeries or experience medical trauma may require rapid reversal of the anticoagulant effect to reduce their risk of bleeding due to anticoagulation [9]. For all reversal agents, the benefits of stopping or reducing bleeding must be balanced against the risk of thrombosis while anticoagulation is reversed $[10,11]$.

Given its aging population-about $27 \%$ of the population in Japan are aged $\geq 65$ years [12]—clinical scenarios requiring anticoagulant reversal are expected to be encountered more frequently in Japan, and this is for two main reasons. Firstly, around one-half of the patients experiencing severe trauma in Japan are aged $>60$ years $\quad(52.9 \%$ of 127,303 trauma patients), with the most frequent cause being ground-level falls (55.2\%) [13]. Emergency surgery for bone fractures was frequent among elderly trauma patients in a study in Japan $(n=631)$; it was reported in $25 \%$ of those aged $65-84$ and in $18 \%$ of those aged $\geq 85$ years [14]. Secondly, anticoagulant usage (warfarin or DOACs) among 8855 adult patients with NVAF in Japan increased between 2006 and 2013 from 20.0 to $49.1 \%$, with a more dramatic increase in the subgroup aged 65-74 years (7.9-59.9\%) [15]. Specific prescription data for DOACs in 2014 suggests that they comprise $\sim 40 \%$ of all anticoagulant prescriptions in patients with NVAF (aged 18-74 years, $n=4380$ ) [16].

What is not yet known is the incidence rate of emergency surgery or bleeding related to trauma or fracture specifically among patients with NVAF taking DOACs in Japan. The aim of the present study was to assess the need for reversal agents in Japanese patients with NVAF on OAC therapy by determining the incidence of emergency surgeries and major bleeding episodes associated with fractures and trauma in this patient population.

\section{METHODS}

\section{Study Design}

This retrospective, non-interventional study was based on data from existing health insurance claims in Japan. The Japanese Medical Data Vision (MDV) clinical database was used. The MDV database contains data from hospital information systems and administrative claims covering more than 12.94 million patients attending 280 acute hospitals as in- or out-patients within secondary medical care blocks in Japan. These hospitals used the Diagnosis Procedure Combination (DPC) case-mix classification system for inpatient reimbursement claims. Approximately 1500 hospitals are designated as DPC hospitals in Japan [17]. The database contains anonymous information from health insurance claims for outpatients, DPC claims for inpatients, prescriptions, operations and medical procedures, hospitalization and results of laboratory tests from some of the participating hospitals [18].

The study protocol was reviewed and approved by the Keio University's Faculty of Pharmacy Ethics Committee for Research involving humans, which also waived the informed consent requirements on October 11, 2017 in accordance with the Ethical Guidelines for Medical and Health Research Involving Human Subjects and the Declaration of Helsinki (1964) and its later amendments. The study was registered with ClinicalTrials.gov (NCT03254147).

OAC-naive adult patients with NVAF who initiated anticoagulant therapy with dabigatran, warfarin, apixaban, rivaroxaban, or edoxaban between March 14, 2011 and June 30, 2016 were included in the study. The main inclusion criteria were: patients (age $>18$ years) with confirmed diagnosis of NVAF (International Classification of Diseases [ICD] 10 I48), having a first prescription (index date) of any one of the OACs (dabigatran, warfarin, rivaroxaban, apixaban, or edoxaban), and having no prescription of OACs for 6 months prior to the index date (baseline period), as well as enrolment in the MDV database for at least 
6 months. The main exclusion criteria were: patients on OAC therapy and patients who underwent dialysis or kidney transplant during the baseline period; patients with atrial flutter, valvular atrial fibrillation (AF), mechanical valve placement, rheumatic $\mathrm{AF}$, and/or mitral valve prolapse/regurgitation/stenosis during the baseline period; patients with a record of deep vein thrombosis or pulmonary embolism within 6 months of AF diagnosis during the baseline period; and patients receiving treatment with two or more OACs at index date.

To calculate the incidence rate of clinical events during OAC treatment, treatment duration was expressed in patient-years of follow-up. The follow-up period was defined from the day after the index date to the date of any OAC treatment discontinuation, end of continuous enrolment in the database, end of the study period, first occurrence of an event of interest, or death, whichever came earlier. On OAC observation time after switching from one OAC to another was included in the primary analysis, and a sensitivity analysis was undertaken that censored for events after the switch.

Patients were stratified on the basis of prescribed OAC and age ( $\leq 64$ years, $65-74$ years and $\geq 75$ years).

\section{Outcomes}

The primary objective of the study was to assess the overall and age-stratified incidence rates of emergency surgery and major bleeding due to fracture and trauma in adult patients initiating OAC therapy for NVAF. Emergency surgery was defined as any surgical procedure (ICD10 code K000-879) performed on the day of hospital admission with additional claims associated with urgent fee MDV codes (114701370, $160000210,180709110)$. Major bleeding due to fracture was defined as any bleeding associated with hospitalization or blood transfusion (ICD10 code K920) in patients with fracture (ICD 10 codes M484, M80, S021, S12, S22, S32, S42, S52, S62, S72, S82, S92, T02, T08, T10, T12, T142) listed as the primary diagnosis (i.e., the diagnosis requiring the most medical resource). Major bleeding due to trauma was defined as any bleeding associated with hospitalization or blood transfusion (ICD10 code K920) in patients with any kind of trauma (ICD 10 codes S00-S09, I620, M125, M164, M165, M172, M173, M191, M483, M872, S065, S066, S081, S089, S130, S18, S230, S480, S481, S532, S533, S580, S581, S589, S633, S634, S680, S681, S684, S688, S689, S780, S789, S889, S980, S981, S982, T050, T053, T058, T059, T116, T136, T147, T794, T796, T797, S271, S272, S330, S334, S382), listed as the primary diagnosis.

The secondary objective of the study was to assess the overall and age-stratified incidence rates of cardiac tamponade and pericardiocentesis. All cardiac tamponade (ICD 10 code 4200001) and pericardiocentesis cases (MDV procedure code 140010510) on the same or next day as catheter ablation or percutaneous coronary intervention (MDV procedure codes 150153910, 150267810, 150263310, 150284310, 150303310, 150345710, 150374910, 150375010, $150375210,150375310,150375410$ ) were included. The types of emergency surgeries were also identified in the study population.

\section{Statistical Analysis}

All MDV data used during the study were in electronic format and were managed by Milliman Inc. Statistical analysis was performed using SAS version 9.4 and Microsoft Excel 2010. Descriptive statistical methods were used for analyzing baseline and outcomes data. The primary outcomes, further outcomes and patient characteristics were determined both in total and in age-stratified groups.

\section{RESULTS}

Within the database, 62,888 patients with NVAF who initiated OAC monotherapy were identified, of whom 53,969 met the criteria for study inclusion (Fig. 1). Overall, $40 \%$ of the study population were female and $60 \%$ of the overall cohort were aged $\geq 75$ years. The mean age \pm SD of the study cohort was $76 \pm 10$ years (Table 1). 


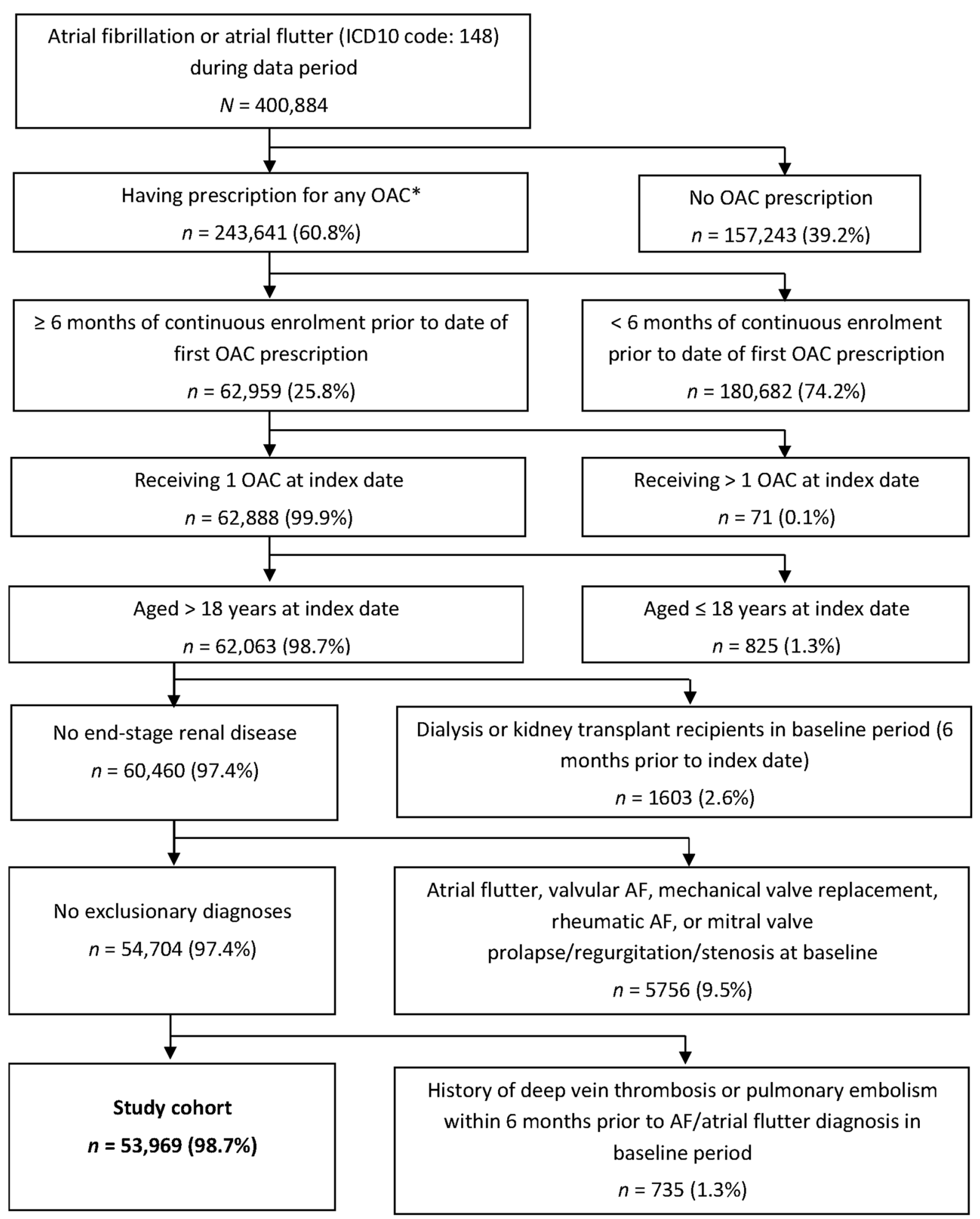

Fig. 1 Patient flow diagram. $A F$ atrial fibrillation, $O A C$ oral anticoagulants. * Between March 14, 2011 and June 30,2016 
Table 1 Baseline patient characteristics

\begin{tabular}{|c|c|}
\hline & $\begin{array}{l}\text { NVAF patients } \\
(n=53,969)\end{array}$ \\
\hline Females, $n(\%)$ & $21,587(40.0)$ \\
\hline Mean \pm SD age, years & $76 \pm 10$ \\
\hline \multicolumn{2}{|l|}{ Age categories, $n(\%)$} \\
\hline$\leq 64$ years & $6960(12.9)$ \\
\hline $65-74$ years & $14,568(27.0)$ \\
\hline$\geq 75$ years & $32,441(60.1)$ \\
\hline \multicolumn{2}{|l|}{ Comorbidities, $\%$} \\
\hline Arterial hypertension & 56 \\
\hline Heart failure & 33 \\
\hline Bleeding & 29 \\
\hline Diabetes mellitus & 24 \\
\hline Dyslipidemia & 22 \\
\hline Valvular disease & 22 \\
\hline Stroke or transient ischemic attack & 11 \\
\hline Peripheral artery disease & 8 \\
\hline Liver disease & 8 \\
\hline Fracture & 5 \\
\hline Dementia & 3 \\
\hline Myocardial infarction & 2 \\
\hline Kidney impairment & 2 \\
\hline Trauma & 2 \\
\hline Nursing home resident & 1 \\
\hline \multicolumn{2}{|l|}{ Concomitant medication, $n(\%)$} \\
\hline Calcium channel blockers & $23,474(43.5)$ \\
\hline Proton pump inhibitor & $21,647(40.1)$ \\
\hline$\beta$-blocker & $19,044(35.3)$ \\
\hline Diuretics & $18,966(35.1)$ \\
\hline $\mathrm{ARB} / \mathrm{ACEI}$ & $17,838(33.1)$ \\
\hline Statins & $11,083(20.5)$ \\
\hline Aspirin & $10,313(19.1)$ \\
\hline $\mathrm{H}_{2}$ receptor antagonist & $8630(16.0)$ \\
\hline Clopidogrel & $4261(8.0)$ \\
\hline
\end{tabular}

Table 1 continued

\begin{tabular}{ll}
\hline & $\begin{array}{l}\text { NVAF patients } \\
(\boldsymbol{n}=53,969)\end{array}$ \\
\hline Amiodarone & $1209(2.2)$ \\
\hline
\end{tabular}

$A C E I$ angiotensin-converting enzyme inhibitors, $A R B$ angiotensin receptor blockers, $S D$ standard deviation

\section{Primary Outcome}

When the follow-up time after switching OAC was included, a total of 27,190 patient-years of follow-up were evaluated in the present study. During follow-up, 133 events of emergency surgery or major bleeding due to fracture or trauma were reported, with the incidence rate calculated as 0.489 per 100 patient-years $(95 \%$ confidence interval [CI] 0.406-0.572). When the follow-up time after switching OAC was excluded, the corresponding figures were 22,972 patient-years and 111 events, with a similar overall incidence rate $(0.483$ per 100 patient-years, 95\% CI 0.394-0.573) (Table 2).

The incidence rates of emergency surgery events or major bleeding events due to fracture or trauma in the subgroup aged $\geq 75$ years (0.611 per 100 patient-years) were almost double those aged $65-74$ years or $\leq 64$ years $(0.388$ and 0.317 per 100 patient-years, respectively) in the analysis that included follow-up after switching OAC. Similar results were noted in the analysis that excluded any follow-up time after switching OAC treatment (Table 2). However, there was some overlap between age groups in the associated $95 \%$ CIs.

\section{Secondary Outcome}

One patient who received warfarin experienced cardiac tamponade and/or pericardiocentesis. Because of the low number of patients who experienced this outcome, the incidence rate was not calculated. 
Table 2 Emergency surgery and major bleeding due to fracture or trauma

\begin{tabular}{|c|c|c|c|c|}
\hline & $\begin{array}{l}\leq 64 \text { years } \\
(n=6960)\end{array}$ & $\begin{array}{l}65-74 \text { years } \\
(n=14,568)\end{array}$ & $\begin{array}{l}\geq 75 \text { years } \\
(n=32,441)\end{array}$ & $\begin{array}{l}\text { Total } \\
(n=53,969)\end{array}$ \\
\hline \multicolumn{5}{|c|}{ Including time after oral anticoagulant switching } \\
\hline Follow-up, patient-years & 4412 & 9028 & 13,750 & 27,190 \\
\hline Patients with any event, $n$ & 14 & 35 & 84 & 133 \\
\hline $\begin{array}{l}\text { IR per } 100 \text { patient-years }(95 \% \\
\text { CI) }\end{array}$ & $\begin{array}{l}0.317(0.151 \\
0.483)\end{array}$ & $0.388(0.259,0.516)$ & $\begin{array}{l}0.611(0.481 \\
0.741)\end{array}$ & $\begin{array}{l}0.489(0.406 \\
0.572)\end{array}$ \\
\hline \multicolumn{5}{|c|}{ Excluding time after oral anticoagulant switching } \\
\hline Follow-up, patient-years & 3700 & 7584 & 11,688 & 22,972 \\
\hline Patients with any event, $n$ & 12 & 28 & 71 & 111 \\
\hline $\begin{array}{l}\text { IR per } 100 \text { patient-years }(95 \% \\
\text { CI) }\end{array}$ & $\begin{array}{l}0.324(0.141 \\
0.508)\end{array}$ & $0.369(0.233,0.506)$ & $\begin{array}{c}0.607(0.467 \\
0.748)\end{array}$ & $\begin{array}{l}0.483(0.394, \\
0.573)\end{array}$ \\
\hline
\end{tabular}

$C I$ confidence interval, $I R$ incidence rate

\section{DISCUSSION}

We have determined that the annual incidence rate of emergency surgery or major hemorrhage associated with fracture and injury was $~ 0.5 \%$ among 53,969 adult NVAF patients on OAC therapy. In the subgroup of very elderly patients (aged $\geq 75$ years) this annual rate was $\sim 0.6 \%$, which was almost double that of those aged $\leq 64$ years $(\sim 0.3 \%)$.

Since our study did not include assessment of OAC-related bleeding, only bleeding related to fractures or trauma/injury and emergency surgery, this may explain why our incidence rates were lower than reported in previous retrospective or observational analyses conducted in adult patients with NVAF receiving OAC therapy (warfarin or DOAC) that reported major bleeding incidence rates (2.4-7.5 per 100 person years [19-21]) or cumulative incidence (1.2-4.7\% [22, 23]).

In addition, OAC-related major bleeding rates may generally be lower among Japanese patients than in patients from other countries, as exemplified by the Global Anticoagulant Registry in the FIELD-Atrial Fibrillation (GARFIELD-AF) ongoing multi-national observational study of stroke prevention in patients with newly diagnosed NVAF [24]. In this study, the major bleeding event rate in Japan was 0.32 (95\% CI 0.19-0.53) per 100 patient-years versus $0.91(0.82-1.00)$ per 100 patient-years in other countries [24]. Major bleeding incidence rates in one Japanese study were 2.2 per 100 patientyears (patients aged $\geq 75$ years) and 1.4 per 100 patient-years (patients aged $<75$ years) among 9578 NVAF patients receiving rivaroxaban [25]. Major bleeding rates associated with DOAC therapy $(n=1676)$ that varied by age were also reported by Nishida and colleagues [26]: 0.973 per 100 patient-years (age $<75$ years), 1.720 per 100 patient-years (age 75-84 years), and 0.985 per 100 patient-years ( $\geq 85$ years). Annualized major bleeding incidence rates in Japanese NVAF patients on warfarin were $1.2 \%$ per year and on a DOAC were $0.5 \%$ per year in an analysis of 3-year clinical outcomes of a Japanese registry study [27]. Another Japanese claims database study reported the incidence rates per 1000 patient-years of major bleeding (6.39), GI bleeding (1.59), and intracranial bleeding (0) among 4606 dabigatran recipients [28].

We did not determine the incidence rate of bleeding associated with fractures or trauma separately from emergency surgery. Falls are the primary cause of severe trauma in the elderly [13]. Falls may lead to traumatic brain injury 
(TBI [29]), traumatic spinal cord injury (TSCI [30]), or fractures. The incidence of falls is higher with increasing age, as is the incidence of fractures. Several Japanese studies have reported an age-related increase in the incidence of ground-level falls resulting in severe trauma [13], annual hip fracture incidence [31], bone fractures requiring emergency surgery in trauma patients [14], annual TSCI incidence [30], and TBI from non-motor vehicle accident causes [32]. While much of these data on falls, fractures, and severe traumatic injury are not specific for patients with NVAF receiving OAC therapy, the age-related increased incidence of such events makes it likely that trauma clinicians and surgeons will encounter patients with NVAF receiving OAC therapy.

Our study has defined the incidence rate of patients undergoing emergency surgery or who have bleeding associated with fractures or trauma in Japan. Together with data indicating that falls are a large contributory factor to the incidence of trauma in Japan [13], that OACrelated bleeding may occur in up to $2.2 \%$ of patients with NVAF, and Japan's aging population, there is a clear reason to anticipate an increased need for anti-coagulation reversal agents.

The main limitation of the present study was the fact that, as a retrospective database analysis, it relied on the integrity of the source information. However, the definitions of outcomes used as endpoints would likely prevent any incorrectly coded events from being included in the analysis, so if anything, is likely to underestimate the incidence of events. Also, given its retrospective nature, it was not possible to capture information about the magnitude of change in hemoglobin levels or the severity of bleeding. As previously mentioned, the current analysis of outcomes was limited to hospitalized or transfused bleeding associated with fracture or trauma, and did not include lifethreatening intracranial or GI bleeding, which were both indications for idarucizumab use in the RE-VERSE AD study [33], or other OAC-related major bleeding. Therefore, this analysis probably underestimates the incidence of bleeding events that may require rapid reversal. Furthermore, the definition of emergency surgery used in the RE-VERSE AD study could not be replicated in this study since the database lacked hourly time stamp information. As a consequence, we used the definition of same day or 1 day after hospitalization, which could result in over-estimation of the incidence rates. Another limitation was that only Japanese patients were included, which could limit the applicability of the findings in other populations. Lastly, we did not conduct any analyses comparing outcomes in patients receiving different types of OACs (warfarin or individual DOACs) or in patients who received reversal agents such as FFP or vitamin K. Since prescribing decisions in real-world clinical practice are individualized for each patient, based on patient demographics and comorbidities, such analyses would be subject to selection and channeling bias that would be difficult to control for statistically, undermining the possibility of obtaining meaningful comparative data.

\section{CONCLUSIONS}

In conclusion, although relatively few patients with NVAF receiving OACs experience emergency surgeries and major bleeding episodes associated with fractures and trauma, the incidence rate of these events is markedly higher in patients of advanced age. This study highlights the importance of having an effective reversal agent for patients on OACs, particularly those in older age groups.

\section{ACKNOWLEDGEMENTS}

We would like to thank Noriyuki Kogo of Milliman Inc. for assistance with statistical analysis of the data (including preparing the plan and conducting the statistical analysis). We would like to thank Hisashi Urushihara for providing advice on the manuscript as well as supporting the IRB approval of the protocol. We would also like to thank Kristina Zint and Lionel Riou Franca for providing valuable advice on the design and manuscript. 
Funding. This project, the statistical analysis, medical writing services, and the journal's rapid service fee were funded by Nippon Boehringer Ingelheim, Japan.

Medical Writing and Editorial Assistance. We would like to thank Yoshiko Okamoto, PhD, Nishad Parkar, PhD and Georgii Filatov of inScience Communications, Springer Healthcare, for writing the partial outline and the first draft of this manuscript. This medical writing assistance was funded by Nippon Boehringer Ingelheim, Japan.

Authorship. All named authors meet the International Committee of Medical Journal Editors (ICMJE) criteria for authorship for this article, take responsibility for the integrity of the work as a whole, and have given their approval for this version to be published.

Disclosures. Masahiro Yasaka received lecture, advisory, and travel fees from Bristol-Myers Squibb, Boehringer Ingelheim, Bayer, CSL Behring and Daiichi Sankyo, as well as scholarship funds or non-restricted grants from Boehringer Ingelheim. Hiroyuki Yokota received lecture or consulting fees from Eisai, Nihon Kohden, Stryker Japan, IMI, Daiichi Sankyo, Asahi Kasei ZOLL Medical, Astellas, Pfizer, Otsuka Pharmaceutical, and Nippon Boehringer Ingelheim. Michiyasu Suzuki received lecture fees from Boehringer Ingelheim and CSL Berling. Teiichi Yamane received lecture or consulting fees from Daiichi-Sankyo, Nippon Boehringer Ingelheim, Abbott Japan, BristolMyers Squibb, Bayer Pharmaceutical Company, and Medtronic Japan. Yasuhisa Ono is an employee of Nippon Boehringer Ingelheim.

Compliance with Ethics Guidelines. The study protocol was reviewed and approved by the Keio University's Faculty of Pharmacy Ethics Committee for Research involving humans, which also waived the informed consent requirements on October 11, 2017 in accordance with the Ethical Guidelines for Medical and Health Research Involving Human Subjects. The study was conducted in compliance with the Declaration of Helsinki (1964) and its later amendments.

Data Availability. The datasets generated during and/or analyzed during the current study are available from the corresponding author on reasonable request.

Open Access. This article is licensed under a Creative Commons Attribution-NonCommercial 4.0 International License, which permits any non-commercial use, sharing, adaptation, distribution and reproduction in any medium or format, as long as you give appropriate credit to the original author(s) and the source, provide a link to the Creative Commons licence, and indicate if changes were made. The images or other third party material in this article are included in the article's Creative Commons licence, unless indicated otherwise in a credit line to the material. If material is not included in the article's Creative Commons licence and your intended use is not permitted by statutory regulation or exceeds the permitted use, you will need to obtain permission directly from the copyright holder. To view a copy of this licence, visit http:// creativecommons.org/licenses/by-nc/4.0/.

\section{REFERENCES}

1. Hart RG, Pearce LA, Aguilar MI. Meta-analysis: antithrombotic therapy to prevent stroke in patients who have nonvalvular atrial fibrillation. Ann Intern Med. 2007;146:857-67.

2. Inoue H, Nozawa T, Hirai T, et al. Accumulation of risk factors increases risk of thromboembolic events in patients with nonvalvular atrial fibrillation. Circ J. 2006;70:651-6.

3. Fuster V, Ryden LE, Cannom DS, et al. 2011 ACCF/ AHA/HRS focused updates incorporated into the ACC/AHA/ESC 2006 guidelines for the management of patients with atrial fibrillation: a report of the American College of Cardiology Foundation/ American Heart Association Task Force on practice guidelines. Circulation. 2011;123:e269-367.

4. De Caterina R, Husted S, Wallentin L, et al. Vitamin $\mathrm{K}$ antagonists in heart disease: current status and perspectives (section III). Position paper of the ESC 
working group on thrombosis-task force on anticoagulants in heart disease. Thromb Haemost. 2013;110:1087-107.

5. Ageno W, Gallus AS, Wittkowsky A, et al. Oral anticoagulant therapy: antithrombotic therapy and prevention of thrombosis, 9th ed: American College of Chest Physicians evidence-based clinical practice guidelines. Chest. 2012;141:e44S-e88S.

6. Hirsh J, Dalen JE, Anderson DR, et al. Oral anticoagulants: mechanism of action, clinical effectiveness, and optimal therapeutic range. Chest. 1998;114:445S-S46969.

7. Heidbuchel H, Verhamme P, Alings M, et al. Updated European Heart Rhythm Association Practical Guide on the use of non-vitamin $\mathrm{K}$ antagonist anticoagulants in patients with non-valvular atrial fibrillation. Europace. 2015;17:1467-507.

8. Yorkgitis BK, Zhang J, Rappold JF. Non-vitamin K antagonist oral anticoagulants: the clinician's new challenge. J Am Osteopath Assoc. 2015;115:612-21.

9. Kaatz S, Kouides PA, Garcia DA, et al. Guidance on the emergent reversal of oral thrombin and factor Xa inhibitors. Am J Hematol. 2012;87(Suppl 1): S141-S145145.

10. Omae T, Koh K, Kumemura M, Sakuraba S, Katsuda Y. Perioperative management of patients with atrial fibrillation receiving anticoagulant therapy. J Anesth. 2019;33:551-61.

11. Thomas S, Makris M. The reversal of anticoagulation in clinical practice. Clin Med (Lond). 2018;18: 314-9.

12. Ministry of Internal Affairs and Communication. Elderly people in Japan-a view from the statistics. 2019. https://www.stat.go.jp/data/topics/pdf/ topics97.pdf. Accessed Sep 2019

13. Kojima M, Endo A, Shiraishi A, Otomo Y. Age-related characteristics and outcomes for patients with severe trauma: analysis of Japan's nationwide trauma registry. Ann Emerg Med. 2019;73:281-90.

14. Nishimura T, Naito H, Matsuyama S, et al. Geriatric trauma in patients $\geq 85$ years old in an urban district of Japan. Acta Med Okayama. 2019;73: 197-203.

15. Kubota K, Ooba N, Kamijima Y, Sato K, Koide D. The use of anticoagulants in patients with nonvalvular atrial fibrillation between 2005 and 2014: a drug utilization study using claims data in Japan. PLoS ONE. 2018;13:e0203380.

16. Ohshima A, Koyama T, Ogawa A, et al. Oral anticoagulants usage in Japanese patients aged 18-74 years with non-valvular atrial fibrillation: a retrospective analysis based on insurance claims data. Fam Pract. 2019;36:685-92.

17. Tanaka S, Seto K, Kawakami K. Pharmacoepidemiology in Japan: medical databases and research achievements. J Pharm Health Care Sci. 2015;1:16.

18. Urushihara H, Taketsuna M, Liu Y, et al. Increased risk of acute pancreatitis in patients with type 2 diabetes: an observational study using a Japanese hospital database. PLoS ONE. 2012;7:e53224.

19. Amin A, Keshishian A, Trocio J, et al. Risk of stroke/ systemic embolism, major bleeding and associated costs in non-valvular atrial fibrillation patients who initiated apixaban, dabigatran or rivaroxaban compared with warfarin in the United States Medicare population. Curr Med Res Opin. 2017;33: 1595-604.

20. Li XS, Deitelzweig S, Keshishian A, et al. Effectiveness and safety of apixaban versus warfarin in non-valvular atrial fibrillation patients in "real-world" clinical practice. A propensity-matched analysis of 76,940 patients. Thromb Haemost. 2017;117:1072-82.

21. Lip GY, Keshishian A, Kamble S, et al. Real-world comparison of major bleeding risk among nonvalvular atrial fibrillation patients initiated on apixaban, dabigatran, rivaroxaban, or warfarin. A propensity score matched analysis. Thromb Haemost. 2016;116:975-86.

22. Lee KT, Chang SH, Yeh YH, et al. The CHA(2)DS(2)VASc score predicts major bleeding in non-valvular atrial fibrillation patients who take oral anticoagulants. J Clin Med. 2018;7:338.

23. Nielsen PB, Skjoth F, Sogaard M, et al. Effectiveness and safety of reduced dose non-vitamin $\mathrm{K}$ antagonist oral anticoagulants and warfarin in patients with atrial fibrillation: propensity weighted nationwide cohort study. BMJ. 2017;356:j510.

24. Koretsune Y, Etoh T, Katsuda Y, et al. Risk profile and 1-year outcome of newly diagnosed atrial fibrillation in Japan-insights from GARFIELD-AF. Circ J. 2018;83:67-74.

25. Kitazono T, Ikeda T, Ogawa S, et al. Real-world outcomes of rivaroxaban treatment in elderly Japanese patients with nonvalvular atrial fibrillation. Heart Vessels. 2020;35:399-408.

26. Nishida T, Okumura Y, Yokoyama K, et al. Oral anticoagulant use and clinical outcomes in elderly Japanese patients: findings from the SAKURA AF Registry. Heart Vessels. 2019;34:2021-30.

27. Okumura Y, Yokoyama K, Matsumoto N, et al. Three-year clinical outcomes associated with 
warfarin vs. direct oral anticoagulant use among Japanese patients with atrial fibrillation-findings from the SAKURA AF registry. Circ J. 2018;82: 2500-9.

28. Koretsune Y, Yamashita T, Yasaka M, et al. Comparative effectiveness and safety of warfarin and dabigatran in patients with non-valvular atrial fibrillation in Japan: a claims database analysis. J Cardiol. 2019;73:204-9.

29. Wong H, Lovett N, Curry N, Shah K, Stanworth SJ. Antithrombotics in trauma: management strategies in the older patients. J Blood Med. 2017;8:165-74.

30. Kudo D, Miyakoshi N, Hongo M, et al. An epidemiological study of traumatic spinal cord injuries in the fastest aging area in Japan. Spinal Cord. 2019;57:509-15.

31. Orimo H, Yaegashi Y, Hosoi T, et al. Hip fracture incidence in Japan: estimates of new patients in 2012 and 25-year trends. Osteoporos Int. 2016;27: $1777-844$.

32. Okuno K. Current status and future of traumatic brain injury treatment in Japan [in Japanese]. Jpn J Neurosurgery (Tokyo). 2018;27:17-24.

33. Pollack CV Jr, Reilly PA, van Ryn J, et al. Idarucizumab for dabigatran reversal-full cohort analysis. N Engl J Med. 2017;377:431-41. 